\title{
Effect of sensitization in US heart transplant recipients bridged with a ventricular assist device: Update in a modern cohort
}

\author{
George J. Arnaoutakis, MD, ${ }^{\mathrm{a}}$ Timothy J. George, MD, ${ }^{\mathrm{a}}$ Arman Kilic, MD, ${ }^{\mathrm{a}}$ Eric S. Weiss, MD, MPH, ${ }^{\mathrm{a}, \mathrm{c}}$ \\ Stuart D. Russell, MD, ${ }^{\mathrm{b}}$ John V. Conte, MD, ${ }^{\mathrm{a}}$ and Ashish S. Shah, MD ${ }^{\mathrm{a}}$
}

\begin{abstract}
Objective: Preformed anti-human leukocyte antigen antibodies have been associated with prolonged wait times and increased mortality in orthotopic heart transplantation. We used United Network for Organ Sharing data to examine panel reactive antibody titers in patients bridged to transplant with left ventricular assist devices.
\end{abstract}

\begin{abstract}
Methods: This was a retrospective review of the United Network for Organ Sharing dataset for all patients bridged to orthotopic heart transplantation with a HeartMate II or HeartMate XVE (Thoratec Corp, Pleasanton, Calif) from January 2004 to December 2009. Patients were primarily stratified by device type and secondarily grouped for comparisons by high ( $>25 \%)$ versus low $(0 \%)$ panel reactive antibody activity (class I and II). Outcomes included survival (30-day and 1-year), treated rejection in the year after orthotopic heart transplantation, and primary graft dysfunction. Cox proportional hazards regression examined 30-day and 1-year survival.
\end{abstract}

Results: A total of 871 patients (56.1\%) received the HeartMate II device, and 673 patients $(43.9 \%)$ received the HeartMate XVE device. Patients with high panel reactive antibody had longer duration on the wait list (205 days [interquartile range, $81-344$ ] vs 124 days [interquartile range, $51-270$ ], $P=.01)$. High panel reactive antibody class II was more common in patients with the HeartMate XVE device (51/547 [9.3\%] vs 42/777 [5.4\%], $P<.001)$. When the entire cohort was examined together, there was no 30-day or 1-year survival difference based on panel reactive antibody activity. Device type did not affect post-orthotopic heart transplantation survival, and panel reactive antibody activity was not associated with worse mortality in Cox regression. Although panel reactive antibody activity did not affect rejection in the year after orthotopic heart transplantation for either device type, high panel reactive antibody class II was associated with higher rates of primary graft dysfunction for both devices $(P<.05)$.

Conclusions: This is the largest modern study to examine the impact of detailed panel reactive antibody information in patients bridged to transplant. High panel reactive antibody levels do not affect drug-treated rejection episodes in the first year post-orthotopic heart transplantation; however, there is an associated higher rate of primary graft dysfunction, regardless of device type. Highly sensitized patients bridged to transplant experience excellent survival outcomes after orthotopic heart transplantation. (J Thorac Cardiovasc Surg 2011;142:1236-45)

Supplemental material is available online.

\footnotetext{
From the Divisions of Cardiac Surgery ${ }^{\mathrm{a}}$ and Cardiology, ${ }^{\mathrm{b}}$ and The Bloomberg School of Public Health, ${ }^{\mathrm{c}}$ The Johns Hopkins Medical Institutions, Baltimore, Md.

Dr Arnaoutakis is the Irene Piccinini Investigator in Cardiac Surgery and Dr George is the Hugh R. Sharp Cardiac Surgery Research Fellow. This research was supported in part by National Institutes of Health Grant 1T32CA126607-01A2 (to G.J.A.) and Health Resources and Services Administration Contract 231-00-0115. The content is the responsibility of the authors alone and does not necessarily reflect the views or policies of the Department of Health and Human Services, nor does mention of trade names, commercial products, or organizations imply endorsement by the US government

Disclosures: Authors have nothing to disclose with regard to commercial support.

Read at the 91st Annual Meeting of The American Association for Thoracic Surgery, Philadelphia, Pennsylvania, May 7-11, 2011.

Received for publication May 2, 2011; revisions received June 23, 2011; accepted for publication July 14, 2011; available ahead of print Aug 12, 2011.

Address for reprints: Ashish S. Shah, MD, Assistant Professor of Surgery, Division of Cardiac Surgery, The Johns Hopkins Hospital, Blalock 618, 600 N. Wolfe St, Baltimore, MD (E-mail: ashah29@jhu.edu).

0022-5223/\$36.00

Copyright (c) 2011 by The American Association for Thoracic Surgery doi:10.1016/j.jtcvs.2011.07.019
}

Past studies have considered left ventricular assist device (LVAD) implantation to be a sensitizing event, resulting in elevations of anti-human leukocyte antibody (HLA) levels. ${ }^{1-4}$ Panel reactive antibody (PRA) levels are frequently used to quantify this sensitization. The Food and Drug Administration approval of LVAD therapy as a bridge to heart transplantation has led to increasing numbers of patients awaiting orthotopic heart transplantation (OHT) with LVADs in place. ${ }^{5}$ Although some studies have cited increased mortality in highly sensitized patients bridged to transplant who undergo transplantation, particularly with older-generation pulsatile flow devices, ${ }^{6-8}$ there are other studies that document equivalent post-OHT survival in sensitized and nonsensitized patients. ${ }^{9}$ Also, patients with high PRA levels undergoing conventional OHT are known to experience longer wait-list time and worse 1-year survival. ${ }^{10}$ However, many of these studies are limited by small sample size and continually evolving laboratory techniques. It is unclear whether the observed outcome differences persist on a national scale among all US patients bridged to transplant in 

Abbreviations and Acronyms
HLA = human leukocyte antibody
HMII = HeartMate II
LVAD $=$ left ventricular assist device
OHT $=$ orthotopic heart transplantation
PGD = primary graft dysfunction
PRA = panel reactive antibody
UNOS $=$ United Network for Organ Sharing
$\mathrm{XVE}=$ HeartMate XVE

the modern era of immunomodulatory therapy. Therefore, we used United Network for Organ Sharing (UNOS) data to examine outcomes among highly sensitized patients bridged to OHT.

\section{MATERIALS AND METHODS \\ Data Source}

The UNOS Standard Transplant Analysis and Research database represents an open cohort of prospectively collected donor specific and followup data from October 1987 to December 2009. The dataset used comprises all US patients undergoing thoracic organ transplantation, with follow-up to June 2010. No patient or center identifiers were included, and the study was granted institutional review board exemption.

\section{Study Design}

This study was a retrospective cohort design, including adult $(>17$ years) patients undergoing primary $\mathrm{OHT}$ as a bridge to transplant with the HeartMate II (HMII) or HeartMate XVE (XVE) device (Thoratec Corp, Pleasanton, Calif) from January 2004 to December 2009. PRA major histocompatibility class is not distinguished before 2004 in the UNOS database, and thus the study began in 2004. Exclusion criteria included incomplete ventricular assist device data, heart-lung transplantation, prior OHT, and missing PRA information. The cohort was stratified according to device type (HMII vs XVE).

\section{Panel Reactive Antibody}

PRA levels closest to the time of transplant were used in all patients. Interventions to desensitize patients and timing of ventricular assist device implant are not available in the UNOS database. Thus, PRA levels at listing and peak PRA levels were not evaluated. Highly sensitized patients were defined as having a PRA greater than $25 \%$, and nonsensitized patients were defined as having a PRA of $0 \%$. Strata of PRA activity were defined according to the following groups: $0 \%$, greater than $0 \%$ to $10 \%$, greater than $10 \%$ to $25 \%$, and greater than $25 \%$. Class I and II PRA levels were first examined separately. Because sensitization has been reported in the pediatric OHT literature as an elevated PRA level regardless of class, we also defined a composite PRA score as the highest PRA level in either class. ${ }^{11}$ Comparisons between high $(>25 \%)$ and low $(0 \%)$ PRA activity were performed for both device types. Additional PRA information not contained within the Standard Transplant Analysis and Research file was provided by UNOS. Specifically, 4 categories of PRA assay methods were identified: (1) cytotoxicity assays, (2) enzyme-linked immunosorbent assays, (3) flow cytometry assays, and (4) other.

\section{Variables Examined and Outcome Measures}

The dataset contains more than 400 preoperative, intraoperative, and postoperative variables. Variables examined included primary diagnosis; demographics (age, sex, race, education, and insurance); pre-OHT mechanical ventilation or intensive care unit admission; comorbidities (diabetes mellitus, percent ideal body weight, serum creatinine levels, and hypertension); and transplant variables (ischemic time, HLA mismatch, transplant year, and wait-list time). In addition, hemodynamic measurements (mean pulmonary artery pressure, pulmonary vascular resistance, and cardiac index) were examined. Donor variables examined were age, race, sex, cytomegalovirus status, diabetes, and cigarette use.

The primary end point was 1-year mortality. Secondary outcomes examined were short term mortality (30-day), rejection requiring treatment within the first year after OHT, primary graft dysfunction (PGD), and drug-treated infection. In the UNOS database, PGD is self-reported and defined by each individual center.

\section{Statistical Analysis}

We compared baseline characteristics between the high versus low PRA groups (class I and II separately) by the Student $t$ test (continuous variables) and chi-square test (categoric variables). Survivals of 30 days, 90 days, and 1 year were estimated using the Kaplan-Meier method, because these time intervals have adequate follow-up in this dataset. The Mantel-Cox log-rank test was used to compare survival estimates according to PRA activity. The entire cohort was analyzed according to the Kaplan-Meier method. Separate Kaplan-Meier analysis was performed for XVE and HMII to assess the impact of device type.

A multivariable Cox proportional hazards regression model estimated risk of death with censoring for death, loss to follow-up, and administrative reasons (end of study period). To construct the multivariable model, independent covariates with potential for confounding were first tested in a univariate fashion. In addition to variables associated with mortality on exploratory analysis $(P<.2)$, those with biological plausibility and previously recognized risk factors were incorporated in a forward and backward stepwise fashion into the multivariable model. The likelihood ratio test and Akaike's information criterion in a nested model approach were used to identify which covariates increased the explanatory power of the model. This method favors more parsimonious models. Because the multivariable model was developed with case-wise deletion, all covariates with greater than $15 \%$ missing data were not included. The final model for 1 -year mortality incorporated the following covariates: recipient age, heart failure cause, race, recipient serum creatinine, recipient serum bilirubin, preOHT infection requiring intravenous antibiotics, preoperative mechanical ventilation, allograft ischemic time, center volume, device type (XVE vs HMII), and PRA activity. Although the model was explored with PRA activity as a categoric variable or incorporating strata of PRA activity, the Akaike's information criterion favored PRA as a categoric variable $(>25 \%)$. Visual inspection of complementary log-log plots and testing of Schoenfeld residuals for each variable confirmed that the assumption of proportional hazards had not been violated.

Means are presented with standard deviations, and medians are presented with their interquartile range. Hazard ratios are displayed with 95\% confidence intervals. Statistical testing was performed using STATA software (version 11; StataCorp LP, College Station, Tex).

\section{RESULTS}

\section{Cohort Statistics}

From 2004 to 2009, 1672 patients underwent OHT as a bridge to transplant with an XVE or HMII device in the UNOS database. In regard to device type, 938 patients $(56.1 \%)$ received an HMII and 734 patients $(43.9 \%)$ received an XVE. PRA information was missing in 128 patients $(7 \%)$; thus, the final cohort consisted of 1544 patients ( $\mathrm{n}=673, \mathrm{XVE} ; \mathrm{n}=871$, HMII). The mean age of the cohort was $52 \pm 12$ years, and $84.8 \%$ were male 
TABLE 1. Distribution of panel reactive antibody levels by class

\begin{tabular}{lcccc}
\hline & \multicolumn{4}{c}{ \% PRA activity } \\
\cline { 2 - 5 } Class & $\mathbf{0} \%$ & $\mathbf{1} \% \mathbf{- 1 0} \%$ & $\mathbf{1 1} \%-\mathbf{2 5} \%$ & $>\mathbf{2 5} \%$ \\
\hline I $(\mathrm{N}=1544)$ & $1038(67.2 \%)$ & $194(12.6 \%)$ & $94(6.1 \%)$ & $218(14.1 \%)$ \\
II $(\mathrm{N}=1324)$ & $1078(81.5 \%)$ & $105(7.9 \%)$ & $48(3.6 \%)$ & $93(7.0 \%)$ \\
Composite PRA & $976(63.3 \%)$ & $212(13.8 \%)$ & $114(7.4 \%)$ & $240(15.6 \%)$ \\
XVE (N = 673) & & & & \\
I & $437(64.9 \%)$ & $91(13.5 \%)$ & $46(6.8 \%)$ & $99(14.7 \%)$ \\
II & $424(77.5 \%)$ & $47(8.6 \%)$ & $25(4.6 \%)$ & $51(9.3 \%)$ \\
Composite & $410(60.9 \%)$ & $93(13.8 \%)$ & $57(8.5 \%)$ & $113(16.8 \%)$ \\
$\quad$ PRA & & & & \\
HMII (N = 871) & & & & \\
I & $601(69.0 \%)$ & $103(11.8 \%)$ & $48(5.5 \%)$ & $119(13.7 \%)$ \\
II & $654(84.2 \%)$ & $58(7.5 \%)$ & $23(3.0 \%)$ & $42(5.4 \%) *$ \\
Composite & $566(65.1 \%)$ & $119(13.7 \%)$ & $57(6.6 \%)$ & $127(14.6 \%)$ \\
$\quad$ PRA & & & & \\
\hline
\end{tabular}

*By chi-square analysis, more patients with the XVE device were highly sensitized to PRA class II.

$(\mathrm{n}=1309)$. Recipient race distribution was white, $70.4 \%$ $(\mathrm{n}=1087)$; African-American, $21.3 \%(\mathrm{n}=329)$; Hispanic, $6.0 \%(\mathrm{n}=92)$; and other, $2.3 \%(\mathrm{n}=36)$. Donor race distribution was white, $70.3 \%(\mathrm{n}=1086)$; African-American, $14.8 \%(\mathrm{n}=228)$; Hispanic, $12.7 \%(\mathrm{n}=196)$; and other, $2.2 \%(\mathrm{n}=34)$. During the study period, 234 patients $(15.2 \%)$ died and 178 patients $(11.5 \%)$ did not survive 1 year. One-year incidence of death was 14.7 deaths $/ 100$ person-years. The median follow-up was 12 months (interquartile range, 5-36). Throughout the study period, the number of adult $\mathrm{OHT}$ recipients with a bridge to transplant increased, with 139 (7.0\% of all OHT) in 2004 and 453 $(20.4 \%$ of all OHT) in 2009.

Class I PRA information was available for 1544 patients and class II for 1324 patients. The breakdown of PRA strata is depicted in Table 1. The majority of patients had a PRA level of $0 \%$ for class I $(67.2 \%)$ and class II $(81.4 \%)$. A minority of patients were highly sensitized (PRA $>25 \%$ ) for class I (14.1\%) and class II (7.0\%). When considering the composite PRA (highest PRA value in either class), 240 patients $(15.6 \%)$ were highly sensitized (PRA $>25 \%)$. There were 163 patients $(10.5 \%)$ who were highly sensitized in one class but not the other. The median PRA for highly sensitized patients $(>25 \%$ PRA) who underwent OHT was $58 \%$ and $51 \%$ for class I and class II, respectively. There were patients with a PRA as high as $100 \%$ in each class who underwent OHT. No differences were detected in PRA class I levels by device type; however, more patients with an XVE device were highly sensitized by PRA class II levels $(P<.01)$. In regard to the type of PRA class I assays performed, cytotoxicity was used in 393 patients $(25.5 \%)$, enzyme-linked immunosorbent assays were used in 125 patients $(8.1 \%)$, flow cytometry was used in 740 patients $(47.9 \%)$, and other methods were used in 286 patients $(18.5 \%)$.

\section{Baseline Characteristics}

There were few baseline characteristics that differed between the XVE and HMII groups. Patients in the XVE group tended to be male and to have a history of hypertension. They were also more likely to be hospitalized before OHT and more frequently required preoperative dialysis and intensive care unit care. There were subtle differences in insurance status between the XVE and HMII groups, but these differences were small. The HMII group experienced more time on the wait list. Race, heart failure cause, and the remaining variables were evenly distributed between the 2 device types and are shown in Table 2.

Differences in patients with PRA $0 \%$ versus highly sensitized patients (PRA $>25 \%$ ) are shown in Table 3, for both class I and II. Many clinical characteristics were similar; however, highly sensitized patients tended to be younger and female. African-American OHT recipients were also more likely to be sensitized. Patients with high class I activity spent a longer time on the wait list, but this difference did not reach significance for class II (Table E1). Baseline characteristics of patients in the composite class exhibited similar patterns (data not shown).

\section{Survival}

When the entire cohort was analyzed together, overall survival at 1 year was $86.7 \%$. When comparing survival by device type, 1-year survival estimates were similar between the XVE and HMII groups (XVE, 87.2\% vs HMII, $85.1 \% ; P=.5$ ). No differences were detected when examining survival based on PRA activity. Patients with high class I PRA activity $(>25 \%)$ had equivalent 30-day and 1-year survival as patients with PRA activity of $0 \%$ (30-day, $95.6 \%$ vs $93.8 \% ; P=.3$; 1-year, $86.7 \%$ vs $84.5 \% ; P=.5)$. Likewise, no differences were found when examining class II PRA activity (30-day, $94.7 \%$ vs $93.7 \% ; P=.5 ; 1$-year, $90.7 \%$ vs $85.4 \% ; P=.3)$. Kaplan-Meier 1-year survival estimates are depicted in Figure 1. A comparison was also made in patients with extremely high PRA class I levels $(>90 \%, \mathrm{n}=43)$ versus PRA of $0 \%$, and no differences were observed for 1-year survival $(87.4 \%$ vs $84.3 \%, P=.9)$. Subgroup analysis was performed in patients with idiopathic cardiomyopathy, and no significant differences in survival were detected for 30-day or 1-year mortality, regardless of PRA activity stratification.

By considering each PRA assay independently, we compared 1-year Kaplan-Meier survival estimates in patients with PRA greater than $25 \%$ versus patients with PRA $0 \%$. We repeated this analysis comparing patients with PRA greater than $90 \%$ versus $0 \%$. Regardless of PRA assay type, there were no significant differences in 1-year survival between sensitized and nonsensitized patients by either stratification ( $>25 \%$ vs $0 \%$, or $>90 \%$ vs $0 \%$ ). 
TABLE 2. Baseline characteristics stratified by device type

\begin{tabular}{|c|c|c|c|}
\hline Variable & $\begin{array}{c}\text { XVE } \\
(N=673)\end{array}$ & $\begin{array}{c}\text { HMII } \\
(\mathbf{N}=\mathbf{8 7 1})\end{array}$ & $\begin{array}{c}P \\
\text { value* }\end{array}$ \\
\hline \multicolumn{4}{|l|}{$\begin{array}{r}\text { Demographics and } \\
\text { comorbidities }\end{array}$} \\
\hline Age, y & $51.9 \pm 10.6$ & $51.7 \pm 12.3$ & .8 \\
\hline Male gender & $599(89 \%)$ & $710(82 \%)$ & $<.001$ \\
\hline Hypertension & $213 / 426(50.0 \%)$ & $86 / 217(39.6 \%)$ & .01 \\
\hline Diabetes & $223 / 661(33.7 \%)$ & $265 / 868(30.5 \%)$ & .2 \\
\hline Creatinine, $\mathrm{mg} / \mathrm{mL}$ & $1.3 \pm 0.6$ & $1.3 \pm 0.8$ & .8 \\
\hline Preoperative dialysis & $38 / 658(5.8 \%)$ & $26 / 852(3.0 \%)$ & $<.001$ \\
\hline $\begin{array}{l}\text { Total bilirubin, } \\
\mathrm{mg} / \mathrm{dL}\end{array}$ & $1.1 \pm 1.8$ & $1.2 \pm 2.2$ & .4 \\
\hline Body mass index & $28.6 \pm 5.2$ & $27.7 \pm 12.8$ & .07 \\
\hline $\begin{array}{l}\text { Idiopathic } \\
\text { cardiomyopathy }\end{array}$ & $241 / 582(41.4 \%)$ & $351 / 723(48.5 \%)$ & \\
\hline $\begin{array}{l}\text { Ischemic } \\
\text { cardiomyopathy }\end{array}$ & $326 / 582(56.0 \%)$ & $355 / 723(49.1 \%)$ & \\
\hline \multicolumn{4}{|l|}{ Race } \\
\hline White & $475(70.6 \%)$ & $612(70.3 \%)$ & .6 \\
\hline Black & $146(21.7 \%)$ & $183(21.0 \%)$ & \\
\hline Hispanic & $40(6.0 \%)$ & $52(6.0 \%)$ & \\
\hline \multicolumn{4}{|l|}{$\begin{array}{l}\text { Insurance and } \\
\text { education }\end{array}$} \\
\hline Private insurance & $386 / 673(57 \%)$ & $466 / 870(53.5 \%)$ & .05 \\
\hline Medicare & $174 / 673(25.8 \%)$ & $267 / 870(30.7 \%)$ & \\
\hline Medicaid & $82 / 673(12.2 \%)$ & $113 / 870(13.0 \%)$ & \\
\hline Other pay & $31 / 673(4.6 \%)$ & $24 / 870(2.8 \%)$ & \\
\hline College education & $245 / 513(47.7 \%)$ & $343 / 680(50.4 \%)$ & .4 \\
\hline \multicolumn{4}{|l|}{$\begin{array}{l}\text { Acuity and pulmonary } \\
\text { function }\end{array}$} \\
\hline $\begin{array}{c}\text { Cardiac index, } \\
\text { L/min/BSA }\end{array}$ & $2.3 \pm 0.7$ & $2.4 \pm 0.7$ & .2 \\
\hline $\begin{array}{l}\text { Mean PA pressure, } \\
\mathrm{mm} \mathrm{Hg}\end{array}$ & $28.7 \pm 10.7$ & $28.1 \pm 10.7$ & .3 \\
\hline PCWP, mm Hg & $19.1 \pm 10.0$ & $18.4 \pm 9.8$ & .2 \\
\hline Hospitalized & $299 / 673(44.4 \%)$ & $238 / 870(27.3 \%)$ & $<.001$ \\
\hline ICU pre-OHT & $147 / 673(21.8 \%)$ & $111 / 870(12.8 \%)$ & $<.001$ \\
\hline $\begin{array}{l}\text { Preoperative } \\
\quad \text { ventilator support }\end{array}$ & $12 / 673(1.8 \%)$ & $10 / 871(1.1 \%)$ & .3 \\
\hline \multicolumn{4}{|l|}{$\begin{array}{l}\text { Donor and transplant } \\
\text { variables }\end{array}$} \\
\hline Age, $y$ & $31.2 \pm 11.8$ & $31.4 \pm 11.4$ & .7 \\
\hline Male gender & $547 / 673(81.3 \%)$ & $675 / 871(77.5 \%)$ & .07 \\
\hline Cigarette use & $144 / 668(21.6 \%)$ & $162 / 860(18.8 \%)$ & .2 \\
\hline Diabetes & $20 / 670(3.0 \%)$ & $23 / 868(2.7 \%)$ & .7 \\
\hline Hypertension & $147 / 673(21.8 \%)$ & $217 / 867(25.0 \%)$ & .1 \\
\hline Creatinine, $\mathrm{mg} / \mathrm{dL}$ & $1.3 \pm 1.0$ & $1.3 \pm 1.0$ & .7 \\
\hline Inotropic support & $378 / 672(56.2 \%)$ & $452 / 865(52.2 \%)$ & .1 \\
\hline CMV mismatch & $172 / 673(25.6 \%)$ & $227 / 871(26.0 \%)$ & .8 \\
\hline HLA mismatch & $348 / 572(60.8 \%)$ & $450 / 785(57.3 \%)$ & .2 \\
\hline Time on wait-list, $\mathrm{d}$ & 118 [43-292] & 155 [68-287] & $<.001$ \\
\hline Ischemic time, $\mathrm{h}$ & $3.4 \pm 1.1$ & $3.3 \pm 1.1$ & .3 \\
\hline
\end{tabular}

$\overline{B S A}$, Body surface area; $P A$, pulmonary artery; $P C W P$, pulmonary capillary wedge pressure; $I C U$, intensive care unit; $C M V$, cytomegalovirus; $H L A$, human leukocyte antigen. $* P$ value from Student $t$ test, chi-square test, or Wilcoxon rank-sum test. Continuous variables with standard deviations (interquartile range for time on wait-list). Categoric variables with whole numbers and percentages.

\section{Multivariable Analysis}

After risk adjustment with Cox multivariable analysis, high sensitization by class I or class II PRA activity was not associated with an increased hazard of 30-day (Table E2) or 1-year mortality (Table 4). For 30-day mortality, African-American race, higher recipient serum creatinine, congenital heart failure cause, preoperative recipient mechanical ventilation, and prolonged allograft ischemic time were all associated with an increased hazard of death. On 1-year mortality analysis, recipient age, African-American race, higher recipient serum creatinine, preoperative infection requiring intravenous antibiotics, and prolonged allograft ischemic time all increased the risk of mortality. Device type, center volume, and PRA activity did not affect the hazard of death for 30-day or 1-year mortality.

\section{Rejection and Complications}

The incidence of drug-treated rejection within the first year after OHT was similar in patients with high class I PRA activity compared with patients with $0 \%$ activity. There were also no differences for patients with high class II PRA activity (Table E1). More than $10 \%$ of the patients in this cohort had missing information in the UNOS database for this outcome; however, there was a trend for high class II activity $(84 / 329,25.5 \%$ vs $13 / 37,35.4 \%$, $P=.2$ ). The occurrence of PGD as coded in the UNOS database was higher in patients with high PRA class I (110/ $1035,10.6 \%$ vs $36 / 218,16.5 \% ; P=.01)$ and patients with high PRA class II activity $(99 / 1075,9.2 \%$ vs $22 / 93$, $23.7 \% ; P<.01)$. Rates of drug-treated infection were unaffected by PRA activity (Table E1).

\section{DISCUSSION}

In this national registry study using UNOS data, highly sensitized patients bridged to OHT with an LVAD have equivalent survival as patients bridged to transplant with low PRA activity. On univariate Kaplan-Meier analysis and in a risk-adjusted multivariable Cox analysis, high PRA activity was not associated with increased 30-day or 1-year survival in patients bridged to transplant. However, highly sensitized patients continue to experience longer wait-list times and have higher rates of PGD, although this definition is poorly characterized in the UNOS database. Given the small absolute differences observed for 1 -year mortality, a study would require an estimated $900 \mathrm{pa}-$ tients in both PRA activity groups to be adequately powered to conclusively determine no effect on mortality. However, achieving greater statistical power than our study would be technically difficult, because this study used the largest national registry available to examine a cohort of patients bridged to transplant.

PRA evaluation in OHT candidates has been used to detect circulating antibodies to a random and broad array of 
TABLE 3. Baseline characteristics stratified by panel reactive antibody class I and class II

\begin{tabular}{|c|c|c|c|c|c|c|}
\hline \multirow[b]{2}{*}{ Variable } & \multicolumn{3}{|c|}{ Class I } & \multicolumn{3}{|c|}{ Class II } \\
\hline & $\begin{array}{c}\text { PRA 0\% } \\
(\mathbf{N}=\mathbf{1 0 3 8})\end{array}$ & $\begin{array}{c}\text { PRA }>25 \% \\
(\mathbf{N}=\mathbf{2 1 8})\end{array}$ & $P$ value* & $\begin{array}{c}\text { PRA 0\% } \\
(\mathbf{N}=\mathbf{1 0 7 8})\end{array}$ & $\begin{array}{c}\text { PRA }>\mathbf{2 5} \% \\
(\mathbf{N}=\mathbf{9 3})\end{array}$ & $P$ value* \\
\hline \multicolumn{7}{|l|}{ Demographics and comorbidities } \\
\hline Age, y & $52.2 \pm 11.5$ & $50.2 \pm 12.0$ & .03 & $52.0 \pm 11.6$ & $49.2 \pm 12.2$ & .02 \\
\hline Female gender & 118/1038 (11.4\%) & $75 / 218(34.4 \%)$ & $<.001$ & $139 / 1078(12.9 \%)$ & $39 / 93(41.9 \%)$ & $<.001$ \\
\hline Hypertension & 201/434 (46.3\%) & $43 / 88(48.9 \%)$ & .6 & $181 / 404(44.8 \%)$ & $21 / 43(48.8 \%)$ & .6 \\
\hline Diabetes & $325 / 1032(31.5 \%)$ & $63 / 215(29.3 \%)$ & .5 & $329 / 1071(30.7 \%)$ & $29 / 91(31.9 \%)$ & .8 \\
\hline Creatinine, $\mathrm{mg} / \mathrm{mL}$ & $1.3 \pm 0.7$ & $1.2 \pm 0.9$ & .4 & $1.3 \pm 0.6$ & $1.1 \pm 0.5$ & .01 \\
\hline Preoperative dialysis & $39 / 1012(3.8 \%)$ & $11 / 215(5.1 \%)$ & .4 & $43 / 1053(4.1 \%)$ & $4 / 90(4.4 \%)$ & .9 \\
\hline Total bilirubin, mg/dL & $1.2 \pm 2.3$ & $1.0 \pm 1.5$ & .4 & $1.2 \pm 2.1$ & $0.9 \pm 0.7$ & .2 \\
\hline Body mass index & $27.9 \pm 5.1$ & $27.8 \pm 5.9$ & 6 & $28.3 \pm 11.8$ & $27.5 \pm 5.3$ & .5 \\
\hline Idiopathic cardiomyopathy & 403/1038 (38.8\%) & $79 / 218(36.2 \%)$ & .5 & $536 / 1078(49.7 \%)$ & $48 / 93(51.6 \%)$ & .9 \\
\hline Ischemic cardiomyopathy & 476/1038 (45.9\%) & $85 / 218(39.0 \%)$ & .06 & $459 / 1078(42.6 \%)$ & $37 / 93(39.8 \%)$ & \\
\hline \multicolumn{7}{|l|}{ Race } \\
\hline White & 768/1038 (74.0\%) & $129 / 218(59.2 \%)$ & $<.001$ & $760(70.5 \%)$ & $52(55.9 \%)$ & .04 \\
\hline Black & $185 / 1038(17.8 \%)$ & $74 / 218(33.9 \%)$ & & $229(21.2 \%)$ & $32(34.4 \%)$ & \\
\hline Hispanic & $61 / 1038(5.9 \%)$ & $10 / 218(4.6 \%)$ & & $63(5.8 \%)$ & $5(5.4 \%)$ & \\
\hline \multicolumn{7}{|l|}{ Insurance and education } \\
\hline Private insurance & $580 / 1037(55.9 \%)$ & $114 / 218(52.3 \%)$ & .1 & $603 / 1077(56.0 \%)$ & $51 / 93(54.8 \%)$ & $<.001$ \\
\hline Medicare & 294/1037 (28.3\%) & $63 / 218(28.9 \%)$ & & $322 / 1077(29.9 \%)$ & $19 / 93(20.4 \%)$ & \\
\hline Medicaid & $131 / 1037(12.6 \%)$ & $27 / 218(12.4 \%)$ & & $131 / 1077(12.2 \%)$ & $16 / 93(17.2 \%)$ & \\
\hline Other pay & $32 / 1037(3.1 \%)$ & $14 / 218(6.4 \%)$ & & $21 / 1077(1.9 \%)$ & $7 / 93(7.5 \%)$ & \\
\hline College education & 402/808 (49.8\%) & $73 / 164(44.5 \%)$ & .2 & $421 / 821(51.3 \%)$ & $34 / 74(46.0 \%)$ & .4 \\
\hline \multicolumn{7}{|l|}{ Acuity and pulmonary function } \\
\hline Cardiac index, $\mathrm{L} / \mathrm{min} / \mathrm{BSA}$ & $2.4 \pm 0.7$ & $2.4 \pm 0.7$ & .8 & $2.3 \pm 0.7$ & $2.4 \pm 0.9$ & .4 \\
\hline Mean PA pressure, $\mathrm{mm} \mathrm{Hg}$ & $28.4 \pm 10.9$ & $28.4 \pm 10.8$ & .9 & $28.5 \pm 11.0$ & $28.6 \pm 11.3$ & .9 \\
\hline PCWP, mm Hg & $18.8 \pm 10.0$ & $18.1 \pm 9.8$ & .4 & $18.8 \pm 10.0$ & $18.4 \pm 9.9$ & .7 \\
\hline Hospitalized & $355 / 1037(34.2 \%)$ & $81 / 218(37.2 \%)$ & .4 & $383 / 1077(35.6 \%)$ & $36 / 93(38.7 \%)$ & .5 \\
\hline ICU pre-OHT & 176/1037 (17.0\%) & $39 / 218(17.9 \%)$ & .7 & 194/1077 (18.0\%) & $19 / 93(20.4 \%)$ & .5 \\
\hline Preoperative ventilator support & $15 / 1038(1.5 \%)$ & $4 / 218(1.8 \%)$ & .7 & $20 / 1078(1.9 \%)$ & $0 / 93(0 \%)$ & .2 \\
\hline \multicolumn{7}{|l|}{ Donor and transplant variables } \\
\hline Age, y & $31.0 \pm 11.3$ & $32.6 \pm 12.3$ & .06 & $31.6 \pm 11.5$ & $30.5 \pm 12.2$ & .4 \\
\hline Male gender & $920 / 1038(88.6 \%)$ & $143 / 218(65.6 \%)$ & $<.001$ & $849 / 1078(78.7 \%)$ & 70/93 (75.3\%) & .4 \\
\hline Cigarette use & $211 / 1026(20.6 \%)$ & $39 / 216(18.1 \%)$ & .4 & $216 / 1070(20.2 \%)$ & $16 / 92(17.4 \%)$ & .5 \\
\hline Diabetes & $25 / 1033(2.4 \%)$ & $9 / 218(4.1 \%)$ & .2 & $34 / 1075(3.2 \%)$ & $5 / 93(5.4 \%)$ & .3 \\
\hline Hypertension & $255 / 1035(24.6 \%)$ & $37 / 218(17.0 \%)$ & .01 & $251 / 1075(23.3 \%)$ & $23 / 93(23.5 \%)$ & .8 \\
\hline Creatinine, $\mathrm{mg} / \mathrm{dL}$ & $1.3 \pm 1.0$ & $1.3 \pm 1.0$ & .8 & $1.3 \pm 0.9$ & $1.2 \pm 0.8$ & .3 \\
\hline Inotropic support & $561 / 1032(54.3 \%)$ & $117 / 217(54.0 \%)$ & .9 & $574 / 1073(53.5 \%)$ & $51 / 93(54.8 \%)$ & .8 \\
\hline CMV mismatch & $629 / 1033(60.9 \%)$ & $115 / 218(52.8 \%)$ & .03 & $290 / 1078(26.9 \%)$ & $15 / 93(16.1 \%)$ & .02 \\
\hline HLA mismatch & $546 / 909(60.1 \%)$ & $97 / 193(50.3 \%)$ & .01 & $572 / 957(59.7 \%)$ & $44 / 83(53.0 \%)$ & .2 \\
\hline Time on wait-list, $\mathrm{d}$ & 124 [51-270] & 205 [81-344] & $<.001$ & 137 [56-280] & 150 [66-279] & .4 \\
\hline Ischemic time, $\mathrm{h}$ & $3.3 \pm 1.1$ & $3.4 \pm 1.1$ & .3 & $3.4 \pm 1.1$ & $3.3 \pm 1.1$ & .2 \\
\hline
\end{tabular}

$\overline{B S A}$, Body surface area; $P A$, pulmonary artery; $P C W P$, pulmonary capillary wedge pressure; $I C U$, intensive care unit; $C M V$, cytomegalovirus; $H L A$, human leukocyte antigen. $* P$ value from Student $t$ test, chi-square test, or Wilcoxon rank-sum test. Continuous variables with standard deviations (interquartile range for time on wait-list). Categoric variables with whole numbers and percentages.

donor lymphocytes. Given the constraints on ischemic time in cardiac transplantation, most patients do not routinely undergo prospective crossmatching. However, some centers use the PRA activity levels to guide which patients should undergo a prospective crossmatch, and positive T-cell crossmatch has been associated with worse 1-year mortality. ${ }^{12}$ Because of past observations that patients with high PRA have worse outcomes after OHT, these patients are commonly only offered local allografts so that prospective crossmatching can be performed. As such, their donor pool is contracted and they experience longer wait-list times.

This study was confined to the years 2004 to 2009, which may explain different findings from studies conducted in the 1990 s. $^{3,4,12-14}$ The differences in wait-list time suggest that sensitized patients are treated differently by transplant centers. It may be that available treatments or improvements in immunomodulatory therapy are responsible for 

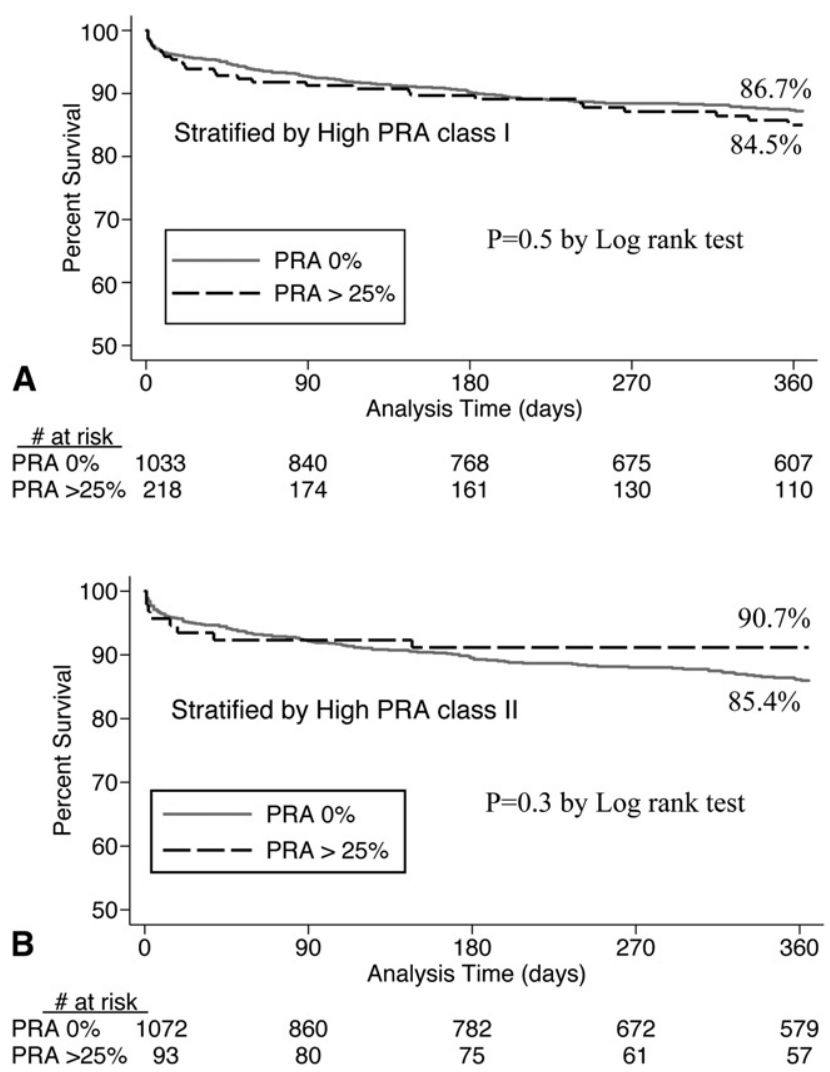

FIGURE 1. Kaplan-Meier estimates of 1-year survival of patients with high versus low PRA class I activity (A) and patients with high versus low PRA class II activity (B). PRA, Panel reactive antibody.

the equivalent outcomes as nonsensitized patients. In the past, sensitization may have been associated with worse outcomes because available therapies were less effective. Moving forward it will be important to understand if the outcomes observed in this study are because PRA levels are less relevant in the current era of immunogenetics testing, immunomodulatory therapy, and perioperative care. If so, this would argue in favor of performing transplants in these patients more aggressively, resulting in shorter wait times. Alternatively, the excellent outcomes in highly sensitized patients bridged to transplant may be due primarily to the current ways in which they are treated, including prolonged wait-list times to undergo desensitization therapy and prospective crossmatching. Information on the percentage of patients who underwent a prospective crossmatch is not well characterized in the UNOS database to better understand this issue.

Stehlik and colleagues ${ }^{15}$ examined the accuracy of a "virtual" crossmatch program and identified a $92 \%$ negative predictive value and $79 \%$ positive predictive value when compared with 257 T-cell antihuman immunoglobulin complement-dependent cytotoxic crossmatch tests. Subsequently, 14 sensitized patients received allografts after a compatible "virtual" crossmatch with donors from distant locations. Their outcomes were compared with 14 sensitized patients who received organs after compatible prospective serologic crossmatch, and similar rejection rates and survival were found. These findings hold promise for improving donor issues in highly sensitized patients, although they warrant duplication in larger-scale studies before widespread use.

There are conflicting reports regarding the impact of device type on formation of anti-HLA antibodies and PRA levels. George and colleagues ${ }^{16}$ evaluated 60 patients who were matched on comorbidities. They detected an increase in PRA post-LVAD insertion with the XVE device but no increase for the HMII device. There were fewer rejection episodes post-OHT in the HMII group in that study, although

TABLE 4. Cox multivariable hazards regression for 1-year mortality

\begin{tabular}{|c|c|c|c|c|c|c|}
\hline \multirow[b]{2}{*}{ Variable } & \multicolumn{3}{|c|}{ Class I* } & \multicolumn{3}{|c|}{ Class II* } \\
\hline & HR & $\mathbf{9 5} \%$ CI & $P$ value & HR & $\mathbf{9 5} \%$ CI & $P$ value \\
\hline $\mathrm{PRA}>25 \%$ & 1.02 & $0.65-1.62$ & .9 & 0.69 & $0.32-1.51$ & .3 \\
\hline Recipient age, $\mathrm{y}$ & 1.02 & $1.00-1.04$ & .02 & 1.02 & $1.00-1.04$ & .05 \\
\hline Idiopathic cardiomyopathy & - & Reference & - & - & Reference & - \\
\hline Ischemic cardiomyopathy & 1.24 & $0.83-1.85$ & .3 & 1.38 & $0.92-2.08$ & .1 \\
\hline Congenital & 1.14 & $0.53-2.44$ & .7 & 1.62 & $0.84-3.10$ & .1 \\
\hline White & - & Reference & - & - & Reference & - \\
\hline African-American & 2.06 & $1.35-3.15$ & $<.01$ & 2.10 & $1.37-3.21$ & $<.01$ \\
\hline Hispanic & 1.18 & $0.54-2.60$ & .7 & 1.68 & $0.83-3.40$ & .1 \\
\hline Recipient serum creatinine, $\mathrm{mg} / \mathrm{mL}$ & 1.32 & $1.16-1.50$ & $<.01$ & 1.20 & $1.08-1.34$ & $<.01$ \\
\hline Recipient serum bilirubin, $\mathrm{mg} / \mathrm{mL}$ & 1.00 & $0.95-1.07$ & .9 & 1.03 & $0.98-1.08$ & .2 \\
\hline Preoperative infection requiring IV antibiotics & 1.51 & $1.02-2.24$ & .04 & 1.67 & $1.12-2.46$ & .01 \\
\hline Preoperative mechanical ventilation & 2.21 & $0.82-5.97$ & .1 & 2.09 & $0.82-5.36$ & .1 \\
\hline Allograft ischemic time, $\mathrm{h}$ & 1.17 & $1.02-1.35$ & .03 & 1.16 & $1.00-1.33$ & .04 \\
\hline High annual center volume $(>12)$ & 0.95 & $0.63-1.59$ & .8 & 0.90 & $0.57-1.42$ & 6 \\
\hline HMII device & 1.14 & $0.80-1.62$ & .5 & 1.19 & $0.83-1.71$ & .3 \\
\hline
\end{tabular}

$H R$, Hazard ratio; $C I$, confidence interval; $I V$, intravenous. *Cox multivariable analysis performed with same risk factors in class I model examining class I PRA activity and class II model examining class II PRA activity. 
survival was not affected. Drakos and colleagues ${ }^{1}$ confirmed these findings in 75 patients bridged to transplant, but in that study only 11 patients received the HMII. In contrast, Kumpati and colleagues ${ }^{17}$ found that device type did not affect sensitization in a large series of 239 patients with an LVAD; however, that study spanned a broader time period and examined other devices in addition to the current most widely used HMII device. ${ }^{17}$

Our study builds on these previous findings because the UNOS database distinguishes between class I and class II PRA activity beginning in 2004 and continuing to the present. Thus, we confined our study to include these years only. When comparing highly sensitized patients, we chose more than $25 \%$ as the cut-point. There is no consensus definition on what threshold defines high sensitization, but most studies use $10 \%$ or $25 \%$. We did examine strata of PRA activity $(0 \%, 1 \%-10 \%, 11 \%-25 \%,>25 \%)$ so as not to lose sensitivity in the analysis, but the multivariable models with the most explanatory power incorporated PRA activity as a categoric variable $(0 \%$ vs $>25 \%)$. To evaluate extremely sensitized patients, patients with PRA greater than $90 \%$ were compared with patients with PRA $0 \%$, and we observed similar findings. In regard to the influence of device type, we detected higher PRA activity in patients with an XVE for class II PRA activity only. Our study in part supports the findings by other investigators, although we were unable to account for pre-LVAD PRA activity.

There were few other baseline differences in characteristics between nonsensitized and sensitized patients. Women were more likely to have high PRA levels, consistent with pregnancy being a sensitizing event. In addition, AfricanAmerican recipients were more likely to have high class I and class II PRA levels. Past studies have documented difficulty with HLA matching and poorer long-term survival in African-American OHT recipients. ${ }^{18,19}$ Although sensitization seems more prevalent in African-American recipients, in our study high PRA levels did not affect post-OHT survival in patients bridged to transplant. Thus, biologic mechanisms other than PRA activity are likely combined with socioeconomic factors to contribute to this outcome disparity.

\section{Multivariable Analysis}

Risk-adjusted multivariable Cox regression analysis assessed the impact of potential confounding variables on 30 -day and 1-year survival. Weiss and colleagues ${ }^{20}$ recently developed a quantitative recipient risk index (IMPACT) for OHT recipients, and many of these variables were selected for inclusion in our multivariable analysis. For 1-year survival, older recipient age, recipient creatinine, AfricanAmerican race, pre-OHT infection, and longer allograft ischemic time all increased the hazard of 1-year mortality. High PRA activity, whether class I or II, did not independently affect the risk of mortality for 30-day or 1 -year mortality. Although the absolute differences were small, the distribution of recipient age, race, and serum creatinine (for class II only) did differ by PRA activity levels. However, these baseline differences should have been accounted for in the multivariable analysis. We examined device type and center volume in the model because these factors have influenced outcomes in other studies. ${ }^{6,21}$

Bull and colleagues ${ }^{6}$ found worse mortality in sensitized patients with idiopathic cardiomyopathy, so we performed a subgroup analysis in patients with this heart failure cause only. ${ }^{6}$ In contrast with their findings, our analysis did not show increased mortality in highly sensitized patients, and device type did not seem to affect the risk of 30-day or 1 -year mortality.

\section{Limitations}

Because of the retrospective study design, we are unable to assert that all possible confounders have been examined. The UNOS dataset is enriched with many variables available for analysis, although it is possible that potentially important variables are not included in this analysis. Furthermore, large registry databases rely on accurate coding. The assumption is that any coding errors or missing data present in the database will occur randomly and thus do not render any bias. If this assumption is incorrect, residual bias may have influenced our conclusions. To mitigate the effects of missing data, we did not incorporate variables with more than $10 \%$ missing data into the regression analysis.

PRA titers are still routinely performed, although with advancements in immunogenetics laboratories, newer methods may replace the PRA activity titer. Currently, there are different assays used in various immunogenetics laboratories, leading to variability in reporting. Although this variability is a limitation of our study, this analysis does incorporate the largest number of patients bridged to transplant undergoing OHT with available PRA information. In addition, the PRA information included in the UNOS database does not permit us to discern baseline levels before LVAD placement. PRA titers are frequently obtained at multiple times during LVAD support, because transfusions, additional surgical procedures, and the presence of infection may dramatically alter the antibody levels. The UNOS database is limited in that we are unable to determine how antibody levels may have changed over the duration of LVAD support and in relation to specific clinical events. The duration of pre-OHT LVAD support and mortality during the pre-OHT support period are unknown. Many patients with high PRA may not be offered transplant and die as a result.

We are also unable to determine specific treatment algorithms that patients with high PRA may have undergone, including desensitizing therapies. Therefore, we chose to 
examine the PRA level immediately before OHT to examine the immunologic status of the recipient just before implantation of the allograft, regardless of prior therapies or PRA levels. It is also impossible to ascertain if a crossmatch was performed retrospectively or prospectively. Furthermore, information on "virtual" crossmatching is not coded in the UNOS database, although this technique is increasingly being used and may enhance donor-recipient matching in the future. Although these data suggest that high PRA does not influence short-term survival, additional information regarding treatment algorithms and crossmatch practices are needed to definitively support a more liberal strategy for patients bridged to transplant with high PRA activity.

This study has a relatively short follow-up period. We chose to begin the study in 2004, because before that time there were few HMII or XVE recipients bridged to transplant in the UNOS registry. Because sensitization may influence chronic rejection, additional follow-up is warranted to determine whether high PRA levels affect longer-term outcomes, such as 5-year survival.

\section{CONCLUSIONS}

This is the largest modern study to examine the impact of detailed PRA information in patients bridged to OHT with an LVAD. High PRA levels do not affect drug-treated rejection episodes in the first year post-OHT; however, there is an associated higher rate of PGD, regardless of device type. Highly sensitized patients bridged to transplant experience excellent short-term survival outcomes after OHT.

\section{References}

1. Drakos SG, Kfoury AG, Kotter JR, Reid BB, Clayson SE, Selzman CH, et al. Prior human leukocyte antigen-allosensitization and left ventricular assist device type affect degree of post-implantation human leukocyte antigen-allosensitization. J Heart Lung Transplant. 2009;28:838-42.

2. DeNofrio D, Rho R, Morales FJ, Kamoun M, Kearns J, Dorozinsky C, et al. Detection of anti-HLA antibody by flow cytometry in patients with a left ventricular assist device is associated with early rejection following heart transplantation. Transplantation. 2000;69:814-8.

3. Massad MG, Cook DJ, Schmitt SK, Smedira NG, McCarthy JF, Vargo RL, et al. Factors influencing HLA sensitization in implantable LVAD recipients. Ann Thorac Surg. 1997;64:1120-5.

4. Stringham JC, Bull DA, Fuller TC, Kfoury AG, Taylor DO, Renlund DG, et al. Avoidance of cellular blood product transfusions in LVAD recipients does not prevent HLA allosensitization. J Heart Lung Transplant. 1999;18:160-5.

5. Miller LW, Pagani FD, Russell SD, John R, Boyle AJ, Aaronson KD, et al. Use of a continuous-flow device in patients awaiting heart transplantation. $N$ Engl $J$ Med. 2007;357:885-96

6. Bull DA, Reid BB, Selzman CH, Mesley R, Drakos S, Clayson S, et al. The impact of bridge-to-transplant ventricular assist device support on survival after cardiac transplantation. J Thorac Cardiovasc Surg. 2010;140:169-73.

7. Joyce DL, Southard RE, Torre-Amione G, Noon GP, Land GA, Loebe M. Impact of left ventricular assist device (LVAD)-mediated humoral sensitization on posttransplant outcomes. J Heart Lung Transplant. 2005;24:2054-9.

8. Smedira NG, Hoercher KJ, Yoon DY, Rajeswaran J, Klingman L, Starling RC, et al. Bridge to transplant experience: factors influencing survival to and after cardiac transplant. J Thorac Cardiovasc Surg. 2010;139:1295-305, 305 e1-4.

9. Pagani FD, Dyke DB, Wright S, Cody R, Aaronson KD. Development of anti-major histocompatibility complex class I or II antibodies following left ven- tricular assist device implantation: effects on subsequent allograft rejection and survival. J Heart Lung Transplant. 2001;20:646-53.

10. Nwakanma LU, Williams JA, Weiss ES, Russell SD, Baumgartner WA, Conte JV. Influence of pretransplant panel-reactive antibody on outcomes in 8,160 heart transplant recipients in recent era. Ann Thorac Surg. 2007;84:1556-63.

11. Jacobs JP, Quintessenza JA, Boucek RJ, Morell VO, Botero LM, Badhwar V, et al. Pediatric cardiac transplantation in children with high panel reactive antibody. Ann Thorac Surg. 2004;78:1703-9.

12. Smith JD, Danskine AJ, Laylor RM, Rose ML, Yacoub MH. The effect of panel reactive antibodies and the donor specific crossmatch on graft survival after heart and heart-lung transplantation. Transpl Immunol. 1993;1:60-5.

13. Lavee J, Kormos RL, Duquesnoy RJ, Zerbe TR, Armitage JM, Vanek M, et al. Influence of panel-reactive antibody and lymphocytotoxic crossmatch on survival after heart transplantation. J Heart Lung Transplant. 1991;10:921-30.

14. Rose EA, Pepino P, Barr ML, Smith CR, Ratner AJ, Ho E, et al. Relation of HLA antibodies and graft atherosclerosis in human cardiac allograft recipients. J Heart Lung Transplant. 1992;11(3 Pt 2):S120-3.

15. Stehlik J, Islam N, Hurst D, Kfoury AG, Movsesian MA, Fuller A, et al. Utility of virtual crossmatch in sensitized patients awaiting heart transplantation. J Heart Lung Transplant. 2009;28:1129-34.

16. George I, Colley P, Russo MJ, Martens TP, Burke E, Oz MC, et al. Association of device surface and biomaterials with immunologic sensitization after mechanical support. J Thorac Cardiovasc Surg. 2008;135:1372-9.

17. Kumpati GS, Cook DJ, Blackstone EH, Rajeswaran J, Abdo AS, Young JB, et al HLA sensitization in ventricular assist device recipients: does type of device make a difference? J Thorac Cardiovasc Surg. 2004;127:1800-7.

18. Allen JG, Weiss ES, Arnaoutakis GJ, Russell SD, Baumgartner WA, Conte JV, et al. The impact of race on survival after heart transplantation: an analysis of more than 20,000 patients. Ann Thorac Surg. 2010;89:1956-64.

19. Park MH, Tolman DE, Kimball PM. The impact of race and HLA matching on long-term survival following cardiac transplantation. Transplant Proc. 1997; 29:1460-3.

20. Weiss ES, Allen JG, Arnaoutakis GJ, George TG, Russell SD, Shah AS, et al. Creation of a quantitative recipient risk Index for Mortality Prediction After Cardiac Transplantation (IMPACT). Ann Thorac Surg. 2011;92:914-22.

21. Weiss ES, Meguid RA, Patel ND, Russell SD, Shah AS, Baumgartner WA, et al Increased mortality at low-volume orthotopic heart transplantation centers should current standards change? Ann Thorac Surg. 2008;86:1250-60.

\section{Discussion}

Dr Carmelo Milano (Durham, NC). Dr Shah and colleagues from Johns Hopkins ought to be commended for their effort to use the UNOS database. A large number of resources are required to maintain the UNOS database. These data have not been extensively analyzed. But recently, Dr Shah and colleagues have published several reviews of these data that have provided us with useful clinical knowledge.

The current study shows that heart transplant recipients who underwent LVAD bridging experienced equivalent posttransplant survival outcomes regardless of device type and PRA status; however, the high PRA group experienced a longer wait-list time relative to the low PRA or zero PRA group. This observation is important and indicates the importance of the pretransplant LVAD support period. I have one slide that I wanted to show.

This slide illustrates survival outcomes from a recent bridged to transplant LVAD device trial. This experimental device was implanted in approximately 140 patients, and the outcomes over the course of 1 year are demonstrated. It is important to note that less than $30 \%$ of the patients actually go on to receive a transplant during the first year of support. So when we talk about bridging patients to transplant, it is a long bridge, with less than $30 \%$ of patients receiving a transplant during the first year.

Furthermore, it is notable that the mortality during device support is less than $5 \%$ in this study, which is actually comparable to 
survival after transplant. Therefore, the prolonged wait-list time may no longer mean that overall survival is reduced for patients with an increased PRA.

My first question is, can the authors display survival outcomes beginning from the time of the LVAD implantation rather than from the time of transplant? The cumulative LVAD and transplant period should be evaluated to determine the impact of factors such as increased PRA or device type on overall survival. Did these factors, increased PRA or the device type, affect outcomes when we look at outcomes beginning from the time of the device implant rather from the time of transplant?

Dr George. One of the great things about the UNOS database is that it contains so many variables. One of the limitations is that the number of variables is finite. Unfortunately, duration of ventricular assist device support is not available. Also, PRA data are only included for patients who ultimately receive transplants. So although there are data on patients who are bridged to transplant who do not ultimately undergo transplantation, we don't actually know what their PRA levels are unless they ultimately receive a transplant. So as you suggest, there is an increased wait-list time, and we would expect that to correlate with an increased wait-list mortality. The data are simply not available in the database to evaluate that.

Dr Milano. My second question relates to posttransplant infection outcomes. Patients with high PRA frequently are treated with different and increased immunosuppressive therapies, for example, plasmapheresis, which may increase infection complications during the posttransplant period. Were the rates of infection different between the 2 groups?

Dr George. We anticipated the infection rate to be higher in the highly sensitized group for that reason. Surprisingly, the infection rates are the same. As measured by class I, nonsensitized patients had an infection rate of approximately $33 \%$ and highly sensitized patients had an infection rate of approximately $35 \%$, and the difference was not significant.

Dr Milano. My third question is, can the authors detect an effect from the advent of virtual crossmatch technology versus formal prospective crossmatch for high PRA LVAD recipients? As we transition to this newer technology, does that seem to be having a positive impact on, for example, wait-list times?

My last question is a more practical, simple question. Many institutions have these LVAD recipients who are highly sensitized. These patients present challenges. We know that their period of LVAD support is prolonged. Given the data that you have presented, has that changed your management? Have you changed your management strategies for these difficult patients on the basis of these data?

Dr George. First, in addressing virtual crossmatch data, we certainly agree that virtual crossmatching is a significant advance in this science. We think it is very important, and it is what we practice at our institution. Unfortunately, whether or not patients underwent virtual crossmatching is another variable that is not available in the database.

In terms of our own practice, it is our practice to use virtual crossmatching, or at least declare at the outset which HLA types will simply be unacceptable for a given recipient. We use prospective crossmatching sparingly, really only for local donors when time will permit. All of our patients undergo retrospective crossmatching, and all of our sensitized patients receive intravenous immunoglobulin on the way to the operating room. We then treat them postoperatively with rituximab, and depending on their crossmatch data, they will get plasmapheresed.

We have always been aggressive about performing transplantations in sensitized patients, because it has been our sense that these patients do well, and I think the results of this study really confirm that practice. So we will continue to be aggressive with highly sensitized patients.

Dr Robert Higgins (Columbus, Ohio). I think the previous questions were important, but I will ask a slightly different question. Did you have any information about prospective crossmatch in this series as it relates to the whole group, because I think it is used in many centers before transplant? And the other question is about prospective management of patients with high PRA, whether they received additional therapies before transplantation. I suspect that may be one of the shortcomings of the UNOS database. But a very nice study.

Dr George. In answering your second question, data relating to desensitization therapies used preoperatively, perioperatively, and postoperatively are not available in the UNOS database. That is something we would certainly be interested in looking at, because it would be interesting to know which treatments are really improving outcomes, and the database in its current form is not able to answer those questions.

My answer is similar in regard to the prospective crossmatching. There is a variable in the database about crossmatching, but there is no designation of prospective versus retrospective. So there is no way to know which patients were prospectively crossmatched and how they did in comparison with those who were only retrospectively crossmatched.

Dr R. Duane Davis (Durham, NC). The idea of using a composite end point that is essentially just the highest of the 2 classes is probably not appropriate given that although they are not independent because of linkage and so forth, it really is closer to an independent variable. So that, in fact, if you were 50\% class I and $50 \%$ class II, then you really are closer to a 75\% PRA. That gets to my question. Did you do any sensitivity analysis around the differing levels of PRA, were there differences in adverse outcomes, particularly at very high levels of PRA?

And then another comment regarding the interpretation of PRA, which doesn't mean the same as having a lot of antibody. It means that you have broad specificities, and it may not be high antibody titers at all.

My final comment will be just a plea to the thoracic community. One of the problems you run into with this data set is that we don't mandate PRA being collected at the time transplant candidacy is done. Therefore, we don't have that data, and we actually can't say what is the impact of being sensitized in terms of dying while waiting for an organ. The only thing we can say is that we have gotten good enough that for the majority of patients we are not having adverse outcomes, at least in the short term.

Dr George. Let me begin by echoing your last comment that the UNOS data set has different tiers of information, the first tier being mandatory reporting and the other tier being voluntary, and it does become difficult in analyzing these data sets when we are missing significant amounts of data in the other tiers. So I do want to echo that comment. 
In addressing the composite end point question, I appreciate those comments. There is an approximately $10 \%$ incidence of patients who are either highly sensitized in one class and not sensitized in the other, and we weren't entirely sure what to do with those patients. And we didn't want to completely ignore this issue and then potentially bias one group or the other, because although they were nonsensitized in one group, they were highly sensitized in another. We wanted some way to take that into account. In the literature we found several articles that used a composite end point such as we have. But I do accept your criticism that that may not be the best way to do this.

The second point about the PRA cut point, any PRA cut point would be somewhat arbitrary in the sense that there are nonsensitized patients, and then there are patients that have some degree of sensitization. Previous literature has suggested different cut points; $10 \%$ and $25 \%$ are 2 commonly used numbers in the literature.

In deciding what cut point to use, we wanted to use a high enough level that we thought the PRA would at least potentially be significant and potentially have an impact on outcomes, and at the same time we wanted to keep the number low enough that we wouldn't lose the power to detect that difference if such a difference existed. So we ended up settling on $25 \%$. However, that said, in our initial analysis and in constructing our models, we did use PRA as a continuous variable. We also stratified it a number of different ways and finally as a dichotomous variable, as we presented. We evaluated our models, and the model with the greatest explanatory power was the one stratified at $25 \%$. But I do again acknowledge the criticism that this is a somewhat arbitrary cut point.

Finally, I want to agree with your point that PRA is sort of a crude measure of sensitization. We recognize that, and we believe that the future of this science lies in specific anti-HLA antibodies and virtual crossmatching. But for now, PRA is the most commonly used measure, so we used it in this study.

Dr Octavio Pajaro (Phoenix, Ariz). Actually I was just going to follow up on Dr Davis's comments. I had similar questions, but with the transition from cell-based assays to solid-based assays and the difference in what we report as a PRA. Really, now we are reporting something that is just the frequency of the allograft in the UNOS population. So it means something different immunologically than it did in 2004.

My question is, can you estimate how unpure your control group is, because especially in hearts, unless you list an unacceptable antigen, then there is no PRA reporting, and many centers won't list anybody with unacceptable antigens. So those patients will appear as a zero PRA on the UNOS, but they are doing a virtual crossmatch.

Dr George. We are limited in this kind of database analysis to what is reported to the database. So we accept that as a limitation of the study, that people may not be entirely truthful about the data. 
TABLE E1. Outcomes stratified by panel reactive antibody level

\begin{tabular}{lrcc}
\hline \multicolumn{1}{c}{ PRA 0 $\%$} & PRA $>\mathbf{2 5} \%$ & $\boldsymbol{P}$ value* \\
\hline Wait-list time, d & & & \\
PRA class I & $123(51-270)$ & $203(81-342)$ & $<.001$ \\
PRA class II & $137(56-280)$ & $151(68-281)$ & .35 \\
Composite PRA & $122(52-266)$ & $200(82-332)$ & $<.001$ \\
Drug-treated rejection & & & \\
PRA class I & $107 / 368(29.0 \%)$ & $25 / 72(34.7 \%)$ & .4 \\
PRA class II & $84 / 329(25.5 \%)$ & $13 / 37(35.1 \%)$ & .2 \\
Composite PRA & $95 / 342(27.8 \%)$ & $31 / 86(36.1 \%)$ & .1 \\
Drug-treated infection & & & .3 \\
PRA class I & $115 / 360(31.9 \%)$ & $28 / 78(35.9 \%)$ & .3 \\
PRA class II & $109 / 323(33.8 \%)$ & $15 / 42(35.7 \%)$ & .9 \\
Composite PRA & $104 / 333(31.2 \%)$ & $34 / 91(37.4 \%)$ & .4 \\
\hline *P value determined by rank-sum analysis for wait-list time and by Pearson chi- \\
square analysis for categoric outcomes.
\end{tabular}

TABLE E2. Cox multivariable proportional hazards regression for 30-day mortality

\begin{tabular}{|c|c|c|c|c|c|c|}
\hline \multirow[b]{2}{*}{ Variable } & \multicolumn{3}{|c|}{ Class I* } & \multicolumn{3}{|c|}{ Class II* } \\
\hline & HR & $95 \%$ CI & $P$ value & HR & $95 \% \mathrm{CI}$ & $P$ value \\
\hline PRA $>25 \%$ & 1.14 & $0.57-2.30$ & .7 & 1.16 & $0.44-3.08$ & .8 \\
\hline Recipient age $>60 \mathrm{y}$ & 1.55 & $0.86-1.54$ & .1 & 1.34 & $0.74-2.42$ & .3 \\
\hline Male recipient & 0.93 & $0.43-2.00$ & .8 & 0.72 & $0.35-1.49$ & .4 \\
\hline Idiopathic cardiomyopathy & - & Reference & - & - & Reference & - \\
\hline Ischemic cardiomyopathy & 1.77 & $0.92-3.44$ & .08 & 2.10 & $1.10-4.01$ & .02 \\
\hline Congenital & 3.22 & $1.29-7.97$ & .01 & 3.30 & $1.42-7.66$ & $<.01$ \\
\hline White & - & Reference & - & - & Reference & - \\
\hline African-American & 2.24 & $1.16-4.30$ & .02 & 2.05 & $1.08-3.92$ & .03 \\
\hline Hispanic & 0.81 & $0.18-3.46$ & .8 & 1.49 & $0.52-4.26$ & .5 \\
\hline Recipient creatinine, $\mathrm{mg} / \mathrm{mL}$ & 1.28 & $1.06-1.54$ & $<.01$ & 1.23 & $1.06-1.43$ & $<.01$ \\
\hline Recipient bilirubin, $\mathrm{mg} / \mathrm{mL}$ & 1.02 & $0.95-1.09$ & .6 & 1.02 & $0.95-1.09$ & 6 \\
\hline Preoperative mechanical ventilation & 4.23 & $1.24-14.46$ & .02 & 4.24 & $1.24-14.46$ & .02 \\
\hline Preoperative infection requiring IV antibiotics & 1.25 & $0.66-2.39$ & .5 & 1.25 & $0.66-2.39$ & .5 \\
\hline HMII device & 1.02 & $0.56-1.80$ & .9 & 1.03 & $0.56-1.80$ & .9 \\
\hline Allograft ischemic time, $\mathrm{h}$ & 1.29 & $1.07-1.56$ & $<.01$ & 1.30 & $1.08-1.56$ & $<.01$ \\
\hline High annual center volume $(>12)$ & 0.77 & $0.34-1.73$ & .5 & 0.77 & $0.34-1.73$ & .5 \\
\hline
\end{tabular}

$H R$, Hazard ratio; $C I$, confidence interval; $I V$, intravenous. *Cox multivariable analysis performed with same risk factors in class I model examining class I PRA activity and class II model examining class II PRA activity. 